\title{
Glucose-regulated protein 78 contributes to the proliferation and tumorigenesis of human colorectal carcinoma via AKT and ERK pathways
}

\author{
XUAN ZHOU $^{1 *}$, XIAOMING XING $^{1 *}$, SHUPING ZHANG ${ }^{2}$, LILI LIU $^{1}$, \\ CHENGQIN WANG ${ }^{1}$, LIN LI ${ }^{1}$, QIUXIA JI ${ }^{1}$ and HUAMIN LIU ${ }^{3}$ \\ ${ }^{1}$ Department of Pathology, Affiliated Hospital of Qingdao University, Qingdao, Shandong 266000; \\ ${ }^{2}$ Department of Obstetrics, Qingdao Municipal Hospital, Qingdao, Shandong 266000; \\ ${ }^{3}$ Department of Oncology, Affiliated Hospital of Qingdao University, Qingdao, Shandong 266000, P.R. China
}

Received February 2, 2016; Accepted July 28, 2016

DOI: 10.3892/or.2016.5097

\begin{abstract}
Glucose-regulated protein 78 (GRP78), a molecular chaperon in the endoplasmic reticulum (ER), is overexpressed in a variety of tumor types and plays a critical role in cancer cell proliferation, migration, invasion and drug resistance. However, the mechanisms underlying the role of GRP78 in tumor carcinogenesis remain largely unknown. In the present study, we found that GRP78 knockdown in colorectal carcinoma (CRC) cells significantly inhibited cell proliferation, colony formation and tumorigenesis in vitro and in vivo. The proliferation inhibition of CRC cells by GRP78 knockdown was associated with an $\mathrm{S}$ phase arrest, a reduced G1/S transition, and a downregulation of phosphorylation of AKT and ERK1/2, key cell cycle regulatory proteins. In addition, GRP78 knockdown enhanced the apoptosis induced by 5-fluorouracil (5-FU) in CRC cells. Taken together, our results indicate that GRP78 plays an important role in the development and progression of CRC and may have therapeutic potential for CRC patients.
\end{abstract}

\section{Introduction}

Colorectal carcinoma (CRC) is one of the most common malignancies worldwide and is a major cause of cancer-related morbidity and mortality $(1,2)$. Although great advances have been achieved in the treatment of CRC over the past few decades, the mean 5-year survival rate is estimated to be less than $10 \%$ when metastasis occurs (3). Currently, the precise

Correspondence to: Dr Xiaoming Xing, Department of Pathology, Affiliated Hospital of Qingdao University, 16 Jiangsu Road, Qingdao, Shandong 266000, P.R. China

E-mail: edithxing@hotmail.com

*Contributed equally

Key words: GRP78, proliferation, tumorigenesis, colorectal carcinoma, AKT, ERK molecular mechanisms of its pathogenesis and progression are still largely unknown.

Glucose-regulated protein 78 (GRP78), also known as immunoglobulin heavy chain-binding protein (BiP) or HSPA5, is a member of heat shock protein 70 (HSP70) family, which is localized in endoplasmic reticulum (ER). GRP78 is a critical regulator of ER function, due to its roles in protein folding and assembly, targeting misfolded protein for degradation, translocation of newly synthesized polypeptides through the ER membrane and ER $\mathrm{Ca}^{2+}$ binding $(4,5)$. ER stress, such as glucose deprivation, hypoxia and oxidative stress, is known to induce glucose-regulated stress response or unfolded protein response (UPR), which results in an accumulation of GRP78 in the ER compartment (6-9). Recently, several studies have demonstrated the overexpression of GRP78 in a variety of solid tumors and GRP78 overexpression contributes to cancer cell proliferation, migration and drug resistance (10-17). Emerging evidence indicates that GRP78 distributes to the cell surface in various tumors and form a complex with specific ligands, exhibiting a wide range of biological effects. Ligation of surface GRP78 by $\alpha 2$-macroglobulin activates Akt-dependent signaling and promotes proliferation and metastasis of prostate cancer cells (18). Isthmin targets cell-surface GRP78 to trigger apoptosis by inducing mitochondrial dysfunction of cancer cells (19). A recent study showed that CRC cells can secrete GRP78, which binds to cell surface GRP78 resulting in the activation of intracellular proliferation signaling (20).

The AKT and ERK signaling pathways regulate many biologic events in cells. Inhibition of these two pathways has been considered as a novel antitumor strategy (21-23). It was suggested that GRP78 oncogenic properties may rely on the activation of AKT and ERK signaling pathways $(13,24)$. Our previous study has revealed that GRP78 is overexpressed in $\mathrm{CRC}$, regulates CRC cell growth and apoptosis and may be an important indicator for malignant transformation (15). However, the exact pathway driven by GRP78 in carcinogenesis remains unclear. In the present study, investigated the role of GRP78 in CRC carcinogenesis using human CRC cells transfected with GRP78 shRNA and elucidated the possible signaling pathway. 


\section{Materials and methods}

Cell culture. The human CRC cell lines RKO and SW620 were purchased from the American Type Culture Collection (ATCC; Manassas, VA, USA). The cells were cultured in RPMI-1640 medium supplemented with $10 \%$ heat inactivated fetal bovine serum (FBS; Gibco, Grand Island, NY, USA). All cells were maintained in a humidified incubator at $37^{\circ} \mathrm{C}$ and $5 \% \mathrm{CO}_{2}$.

Plasmid construction and cell transfection. Lentivirus with the GRP78 shRNA plasmid expression vector (pLV-GRP78 shRNA) and empty vector (pLV-control) were purchased from Shanghai GeneChem Corporation (Shanghai, China). Transfection of pLV-GRP78 shRNA or pLV-control into RKO and SW620 cells was performed using Lipofectamine 2000 according to the manufacturer's instructions. The transfection efficiency was monitored by fluorescence microscopy and the stably transfected cells were selected by G418 at a concentration of $1 \mathrm{mg} / \mathrm{ml}$.

3-(4,5-Dimethylthiazol-2-yl)-2,5-diphenyltetrazolium bromide (MTT) and colony formation assays. After transfection, cells were plated at 4,000 cells/well into 96-well plates and cultured for 1-5 days. MTT reagent (Amresco, Solon, OH, USA) was added into wells on the indicated day, and the cells were incubated for another $4 \mathrm{~h}$ at $37^{\circ} \mathrm{C}$. The supernatants were then removed, and the formazan crystals were dissolved in dimethyl sulfoxide (DMSO) $(150 \mu \mathrm{l} /$ well). The absorbance at $490 \mathrm{~nm}$ for each sample was measured using a multilabel plate reader (PerkinElmer, Waltham, MA, USA). For the colony formation assay, 500 cells were placed in 6-well plates and maintained in complete medium for 2 weeks. Colonies were fixed with methanol, stained with $0.1 \%$ crystal violet and counted.

Cell cycle analysis. RKO and SW620 cells were synchronized via serum starvation for $12 \mathrm{~h}$. Cells were harvested, washed with ice-cold phosphate-buffered saline (PBS) and fixed in $70 \%$ ethanol at $4^{\circ} \mathrm{C}$ overnight. After washed twice with icecold PBS, the fixed cells were stained with $50 \mu \mathrm{g} / \mathrm{ml}$ propidium iodide (Sigma-Aldrich, Saint-Quentin Fallavier, France) in PBS containing 0.1\% Triton X-100 (Sangon, Shanghai, China) and $50 \mu \mathrm{g} / \mathrm{ml}$ RNase A (Beyotime, Shanghai, China) for $30 \mathrm{~min}$ at $37^{\circ} \mathrm{C}$ in the dark. The cell cycle distributions were measured using FACSCalibur flow cytometer (Beckman Coulter, Brea, CA, USA); 10,000 events were collected and assayed for each determination.

Transwell migration and invasion assays. A total of $1 \times 10^{5}$ transfected cells were resuspended in $200 \mu 1$ serum-free medium and placed in the upper compartment of a Transwell chamber (24-well insert, pore size, $8-\mu \mathrm{m}$; Corning Inc., Corning, NY, USA). The lower chamber was filled with $15 \%$ FBS as a chemoattractant and incubated for $24 \mathrm{~h}$ for the migration assay and $24 \mathrm{~h}$ for the invasion assay. For the invasion assay, the inserts were pre-coated with extracellular matrix gel (BD Biosciences, Sparks, MD, USA). After incubation, the cells on the upper surface of the membrane were removed, and the cells on the lower surface were fixed and stained with $0.1 \%$ crystal violet. Five visual fields of each insert were randomly chosen and counted under a light microscope.
Cellular chemosensitivity to 5-fluorouracil (5-FU). RKO and SW620 cells $\left(1 \times 10^{4}\right)$ expressing scramble control or GRP78 shRNA were suspended in $500 \mu \mathrm{l}$ of serum-free RPMI-1640 medium and were seeded into 6-well plates. Twelve hours after seeding, cells were treated with 5-FU at the final concentration of $20 \mu \mathrm{g} / \mathrm{ml}$. The cells were harvested at the time point of 1,6 , 12 or $24 \mathrm{~h}$ for the western blot assay.

Western blotting. Cells were lysed in RIPA buffer (Beyotime) supplemented with protease inhibitor cocktail and phosphatase inhibitors (Aidlab Biotechnologies, Beijing, China). Total protein $(40 \mu \mathrm{g})$ was separated on $10 \%$ SDS-PAGE gel and transferred onto polyvinylidene fluoride (PVDF) membranes. After blocked with Tris-buffered saline with Tween-20 (TBST) containing 5\% fat-free milk for $1 \mathrm{~h}$, the membranes were incubated with the primary antibodies against GRP78 (1:250 dilution; Abcam, Cambridge, MA, USA), AKT (1:1,000 dilution), phospho-AKT (ser473) (1:000 dilution), ERK (1:000 dilution), phospho-ERK1/2 (1:1,000 dilution) (all from Cell Signaling Technology, Danvers, MA, USA) and $\beta$-actin (1:8,000; Santa Cruz Biotechnology, Santa Cruz, CA, USA) at $4^{\circ} \mathrm{C}$ overnight. The membranes were then incubated with secondary incubation using a horseradish peroxidase-conjugated anti-rabbit or anti-mouse IgG (ZSGB-BIO, Beijing, China). After washing, the blots were developed by chemiluminescent reagent (Thermo Scientific, Waltham, MA, USA).

Tumorigenicity assay in nude mice. Five-week-old nude BALB/c athymic strain mice were purchased from Shanghai GeneChem Corporation. Animal operation was carried out according to the protocols approved by the Committee on the Use of Live Animals in Teaching and Research (CULATR). License to conduct live animal experiments was approved by Independent Ethics Committee of Affiliated Hospital of Qingdao University. Animals were subcutaneously injected in the right flank with $4 \times 10^{6} \mathrm{RKO}$ cells expressing scramble control or GRP78 shRNA. Tumor growth was examined every 3 days for 6 weeks. Tumor volume (V) was monitored by measuring the length $(\mathrm{L})$ and width $(\mathrm{W})$ of the tumors and calculated with the following equation: $\mathrm{V}=\left(\mathrm{L} \mathrm{x} \mathrm{W}^{2}\right) \times 0.5$.

Statistical analysis. All experiments were repeated at least 3 times. All data were expressed as the mean \pm SD. Statistical analyses were performed using SPSS 17.0 and 3 group data comparison was assessed by one-way analysis of variance (ANOVA). LSD test was performed for two group comparison. Differences were considered significant for P-values $<0.05$.

\section{Results}

Knockdown of GRP78 expression inhibits the proliferation and colony formation of CRC cells. To determine the involvement of GRP78 in the proliferation and colony formation of CRC cells in vitro, we knocked down the GRP78 in RKO and SW620 cells using specific shRNA. As shown in Fig. 1, GRP78 shRNA transfection markedly decreased the expression of GRP78 in RKO and SW620 cells (Fig. 1A). By MTT assay, the cell proliferation was significantly lower in both RKO and SW620 cells transfected with pLV-GRP78 shRNA than cells transfected with pLV-control $(\mathrm{P}<0.05$; Fig. 1B). Supporting 
A

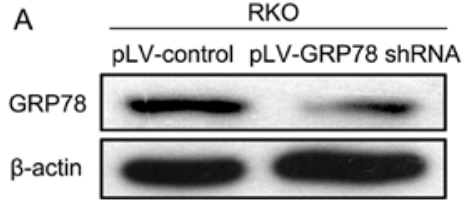

B
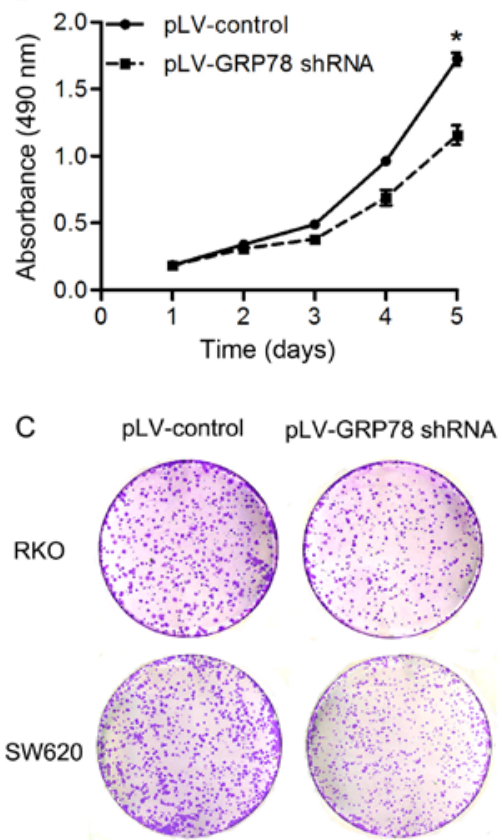

SW620

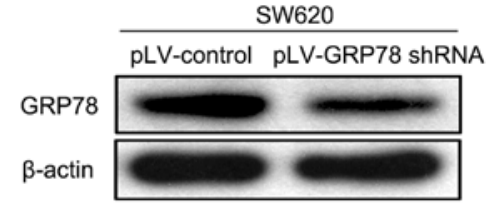

SW620

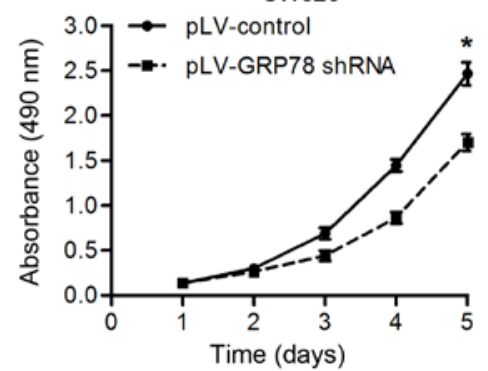

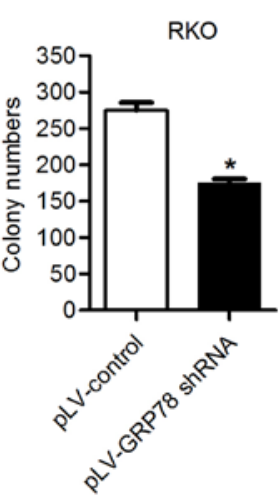

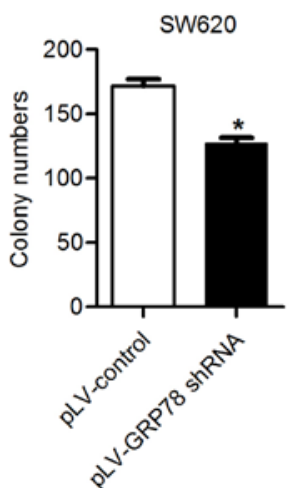

Figure 1. Knockdown of GRP78 expression inhibits the proliferation and colony formation of CRC cells. The endogenous GRP78 expression levels were detected by western blotting in RKO and SW620 cells transfected with pLV-GRP78 shRNA or pLV-control for 24 h. (B) MTT cell viability assays were performed on days 1-5 after the transfection of RKO and SW620 cells with either pLV-GRP78 shRNA or pLV-control. The data represent the mean \pm SD of 3 independent experiments. ("P<0.05 compared with control). (C) The colony formation assays were performed in RKO and SW620 cells after they were transfected with pLV-GRP78 shRNA or pLV-control and incubated for 14 days. The data represent the mean $\pm \mathrm{SD}$ of 3 independent experiments $($ "P $<0.05$ compared with the control).
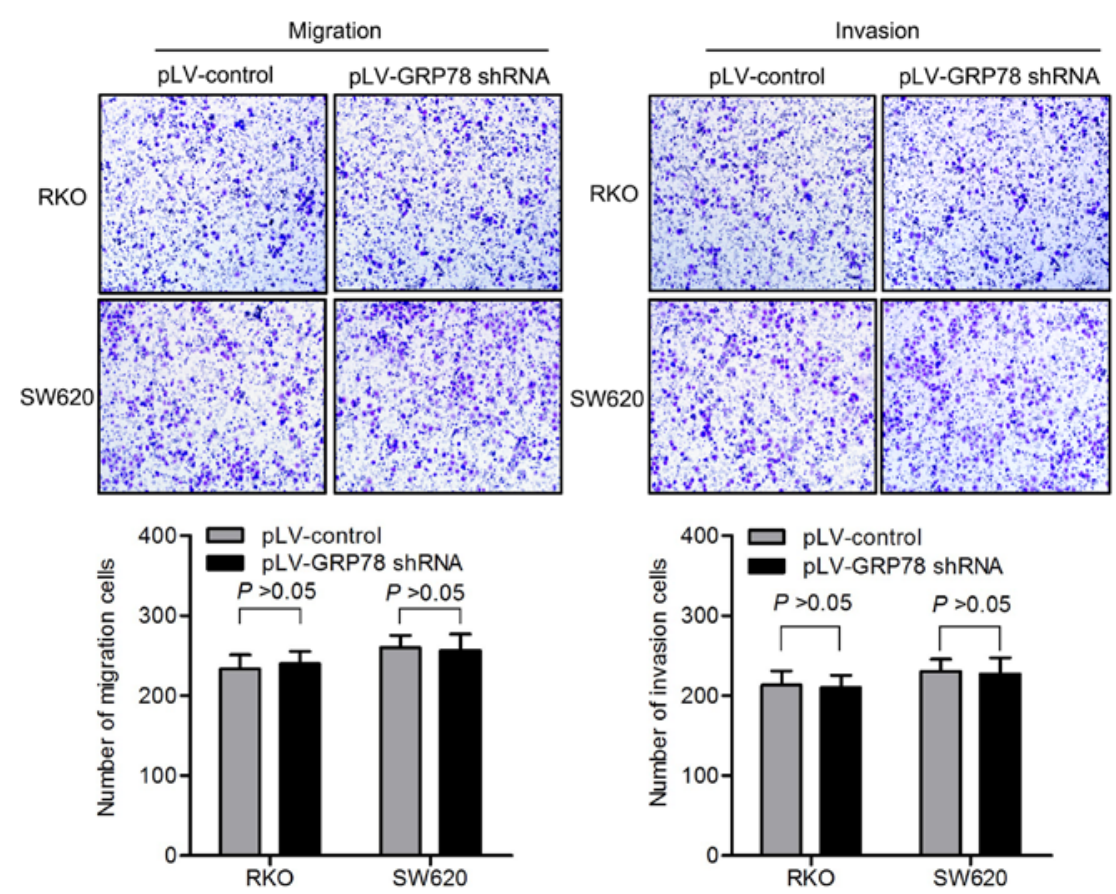

Figure 2. Knockdown of GRP78 had no impact on migration and invasion of CRC cells. The effects of GRP78 on cell migration and invasion were determined by Transwell migration and invasion assays in RKO and SW620 cells transfected with pLV-GRP78 shRNA or pLV-control. 
A
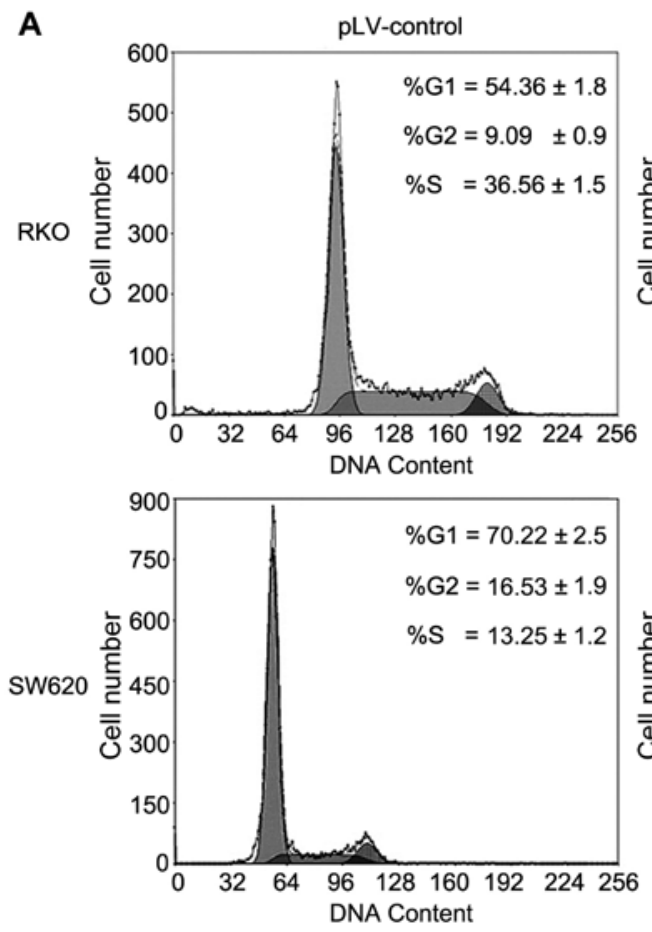

B

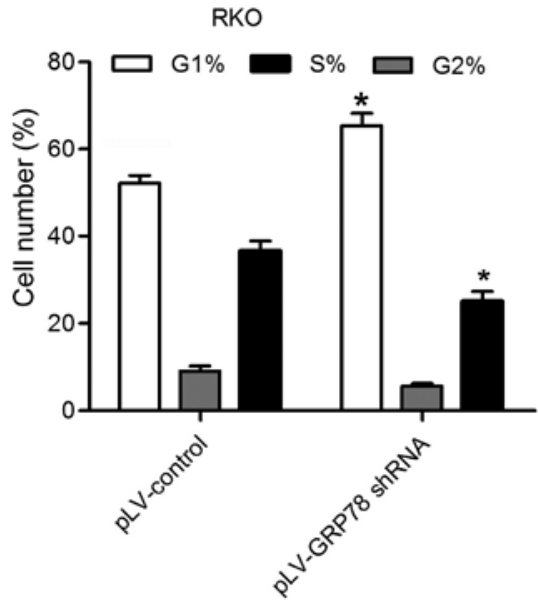

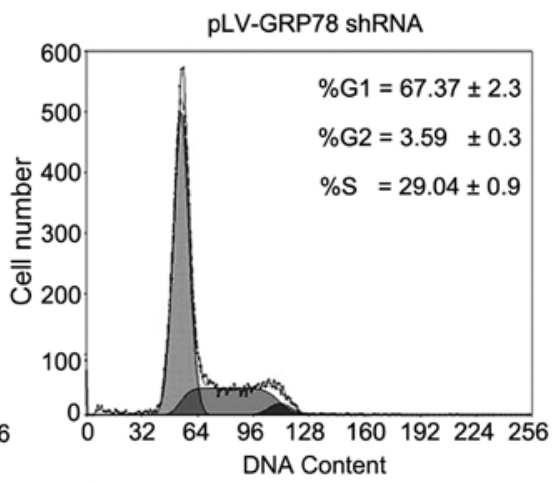
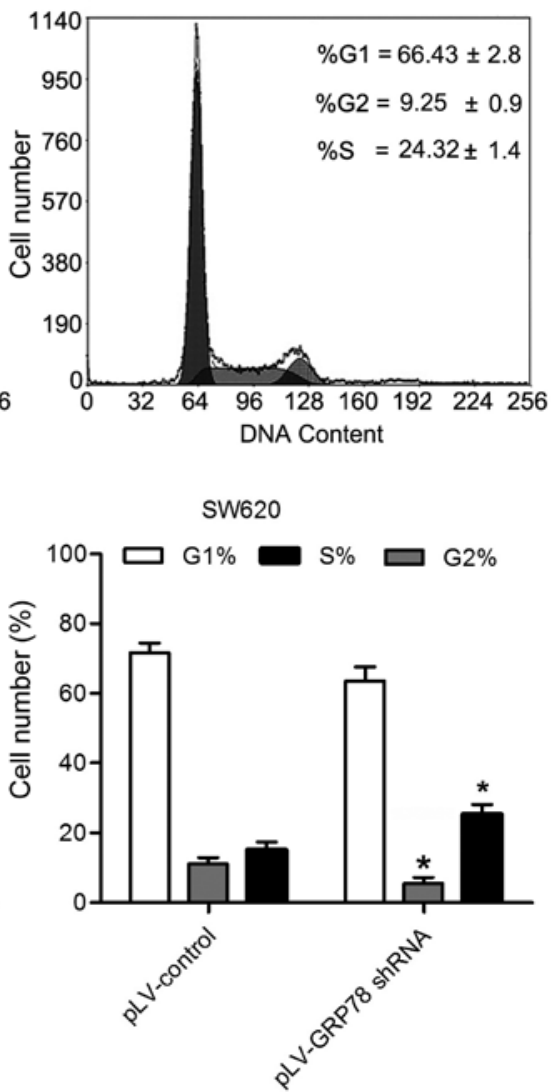

Figure 3. Knockdown of GRP78 inhibits G1/S transition and induces S phase arrest. (A and B) Cell cycle profiles were analyzed by flow cytometer after RKO and SW620 cells were transfected with pLV-GRP78 shRNA or pLV-control for $12 \mathrm{~h}$. Data are from 3 independent experiments and expressed as means \pm SD. ( $\mathrm{P}<0.05$ compared with the control).

this finding, we found that knockdown of GRP78 inhibited the colony formation of both RKO and SW620 cells in vitro. The number of colonies formed in matrix gel was significantly lower in cells transfected with pLV-GRP78 shRNA than cells transfected with pLV-control $(\mathrm{P}<0.05$; Fig. 1C). These results suggest that GRP78 promotes the proliferation and colony formation of CRC cells. The knockdown of GRP78 had no impact on migration and invasion of CRC cells in vitro (Fig. 2).

Knockdown of GRP78 inhibits G1/S transition and induces $S$ phase arrest. To explore the mechanism underlying growth promotion by GPR78, we determined the cell cycle of RKO and SW620 cells by flow cytometry. As shown in Fig. 3, the percentage of cells in G0/G1 phase was increased in RKO cells transfected with pLV-GRP78 shRNA when compared to cells transfected with pLV-control. In contrast, the percentage of cells in S and G2/M phase was significantly decreased in RKO cells transfected with pLV-GRP78 shRNA $(\mathrm{P}<0.05)$. Notably, the percentage of cells in S phase was increased in SW620 cells transfected with pLV-GRP78 shRNA when compared to cells transfected with pLV-control, and G2/M phase was significantly decreased in SW620 cells transfected with pLV-GRP78 shRNA $(\mathrm{P}<0.05)$. These results suggest that knockdown of GPR78 was able to inhibit G1/S transition and induce $S$ phase arrest, consistent with the finding that GRP78 promoted the proliferation of CRC cells.

GRP78 knockdown enhances the chemotherapy sensitivity induced by 5-FU. 5-FU is an effective drug which is widely used in the CRC chemotherapy. In order to evaluate whether GRP78 knockdown had effect on the chemotherapy sensitivity of 5-FU, RKO and SW620 cells were treated with $20 \mu \mathrm{g} / \mathrm{ml}$ 
A
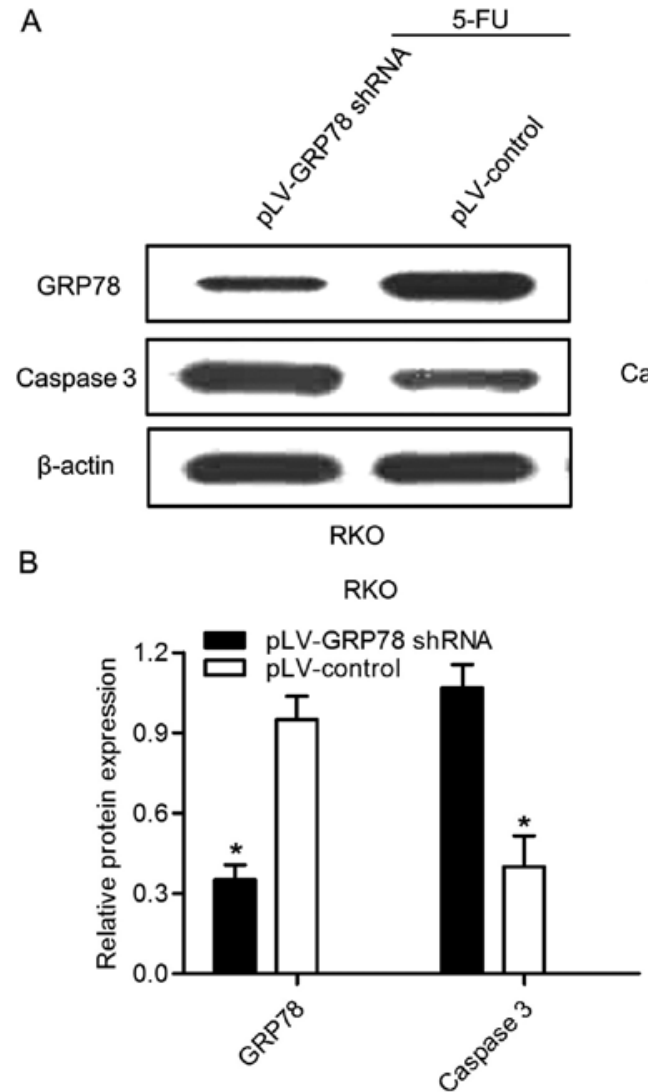
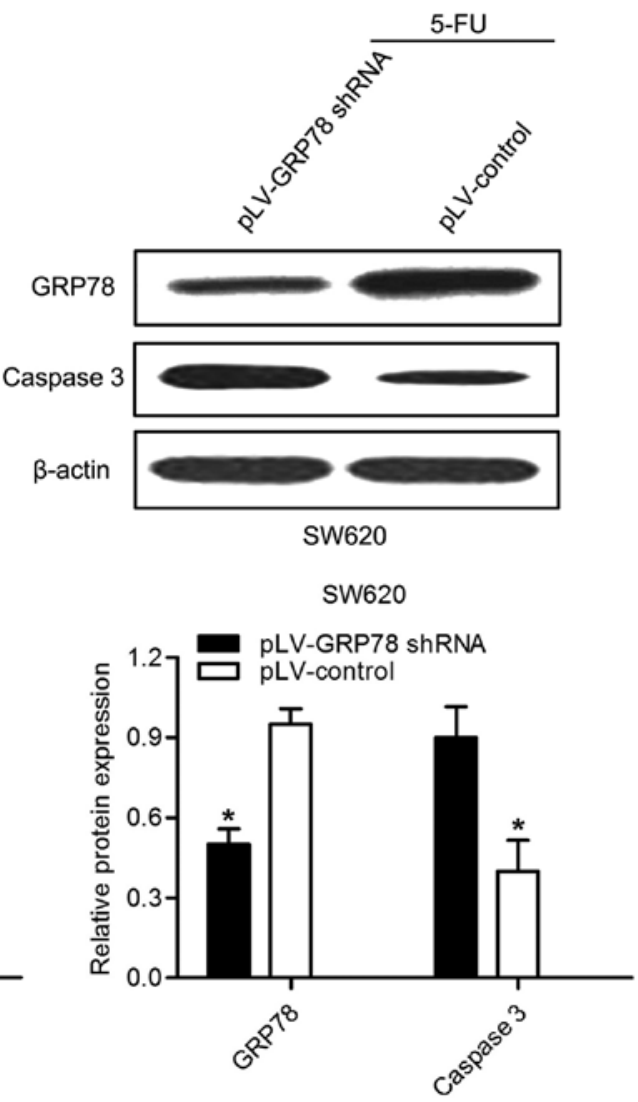

Figure 4. GRP78 knockdown enhances the chemotherapy sensitivity induced by 5-FU. (A and B) The expression level of GRP78 and caspase 3 was detected by western blotting in RKO and SW620 cells treated with $20 \mu \mathrm{g} / \mathrm{ml} 5$-FU for $24 \mathrm{~h}$ after transfection with pLV-GRP78 shRNA or pLV-control. With $\beta$-actin as the internal control. ( $\mathrm{P}<0.05$ compared with the control).

A

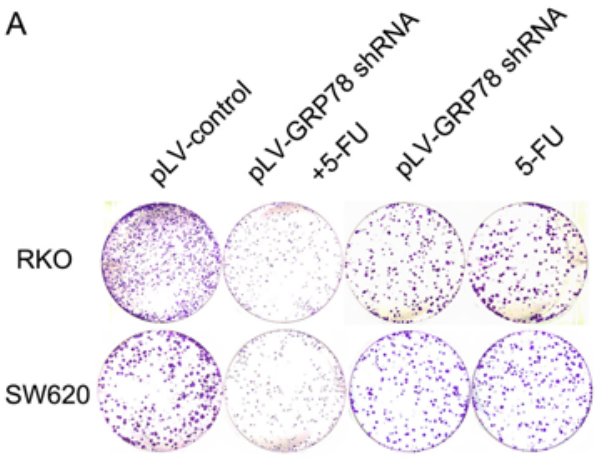

B

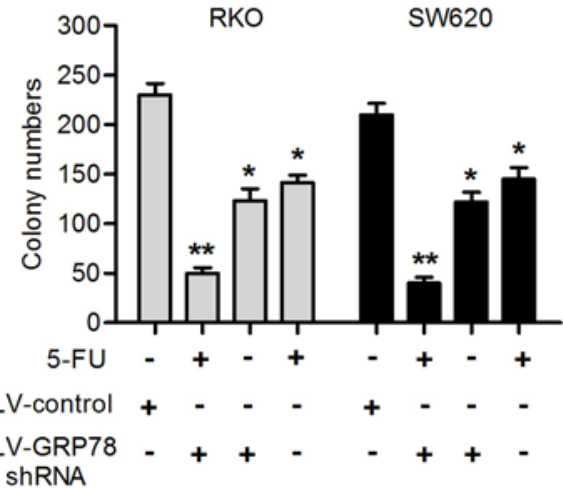

Figure 5. (A and B) The colony formation assays were performed in RKO and SW620 cells transfected with pLV-GRP78 shRNA, pLV-control or treated with $20 \mu \mathrm{g} / \mathrm{ml} 5$-FU for $24 \mathrm{~h}$ after transfection with pLV-GRP78 shRNA or $\mathrm{pLV}$-control and incubated for 2 weeks. The data represent the mean \pm SD of 3 independent experiments ( $\mathrm{P}<0.05,{ }^{* *} \mathrm{P}<0.01$ compared with the control).
5-FU for 24 h. Notably, as shown in Fig. 4, the expression of caspase 3 was significant increased in both cells after GRP78 knockdown. Given that caspase 3 plays a critical role in the regulation of cell death, this result indicates that GRP78 may be able to inhibit the death and apoptosis of CRC cells. In addition, we also found that GRP78 knockdown significantly reduced the number of RKO and SW620 cell colonies after the cells were treated with 5-FU for $24 \mathrm{~h}$ by colony formation assays $(\mathrm{P}<0.05$; Fig. 5). This result suggest that GRP78 may contribute to the therapeutic resistance to 5-FU in RKO and SW620 cells.

Effects of GRP78 knockdown on the phosphorylation of AKT and ERK1/2. To reveal the potential molecular mechanism by which GRP78 promotes the proliferation of CRC cells, we examined phosphorylation of AKT and ERK1/2 by western blot analysis. As shown in Fig. 6, we found that GRP78 knockdown resulted in a downregulation of phosphorylation of both AKT and ERK1/2 in RKO and SW620 cells. These results suggest that GRP78 is involved in the regulation of phosphorylation of AKT and ERK in CRC cells.

Inhibition of GRP78 suppresses tumor growth in vivo. We observed that GRP78 inhibition suppresses the proliferation of CRC cells in in vitro system, we wondered whether GRP78 has a similar effect in in vivo model. To perform this, we inoculated athymic BALB/c mice with RKO cells stably transfected with pLV-GRP78 shRNA or pLV-control and monitored tumor 
A
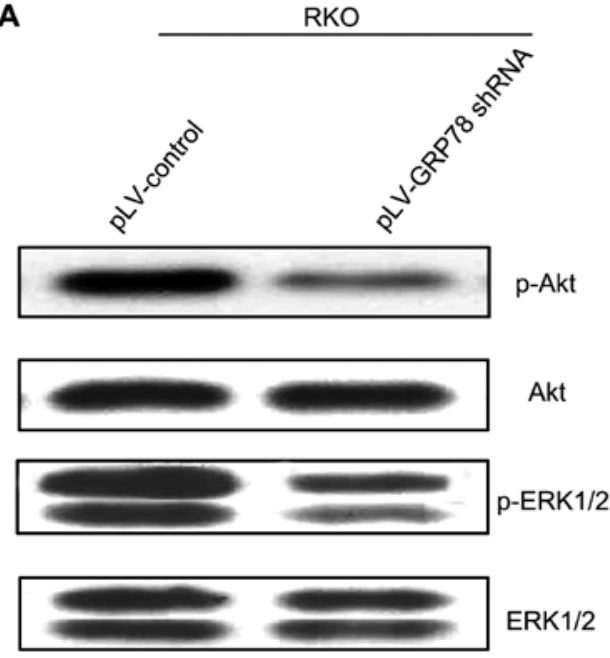

ERK1/2

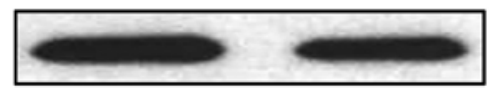

$\beta$-actin

B

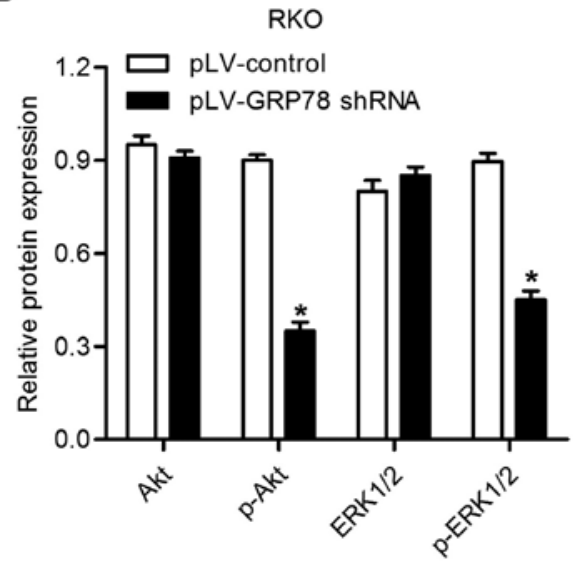

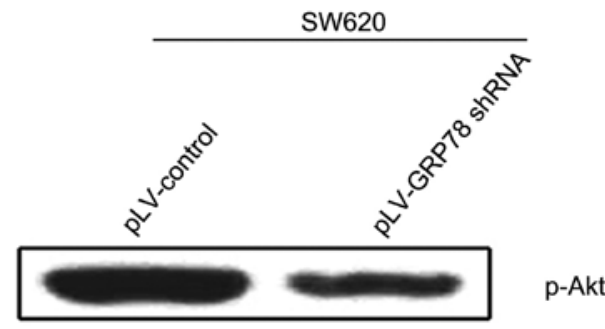

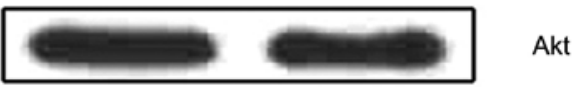

Akt

p-ERK1/2

ERK1/2

$\beta$-actin

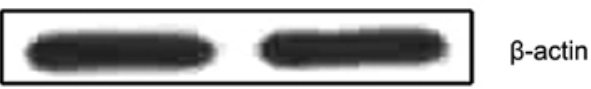

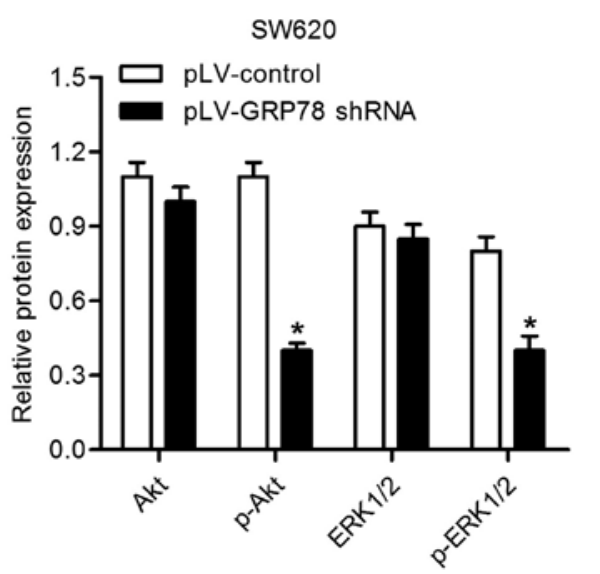

Figure 6. Effects of GRP78 knockdown on the phosphorylation of AKT and ERK1/2. (A and B) The expression level of Akt, p-Akt, ERK1/2 and p-ERK1/2 were detected by western blotting in RKO and SW620 cells transfected with pLV-GRP78 shRNA or pLV-control for $24 \mathrm{~h}$. With $\beta$-actin as the internal control. $($ ( $\mathrm{P}<0.05$ compared with the control).

growth by measuring tumor volume every 3 days for 6 weeks. As shown in Fig. 7A-D, we found that tumors grew at a slower rate with smaller sizes in mice injected cells transfected with pLV-GRP78 shRNA than in those transfected with pLV-control. These results indicate that inhibition of GRP78 suppresses tumor growth in vivo, confirming our finding from in vitro experiments.

\section{Discussion}

The microenvironment of a solid tumor is characterized by glucose deprivation, acidosis and severe hypoxia, all of which lead to the induction of GRP78 $(25,26)$. In the present study, we explored the potential pathways driven by GRP78 in carcinogenesis of colorectal carcinoma (CRC).

Our previous study revealed that GRP78 regulates CRC cell growth and apoptosis and may be an important indicator for malignant transformation. In the present study, we found that silencing GRP78 can suppress the proliferation of CRC cells through inducing $S$ phase arrest and regulating G1/S transition. These findings indicated that GRP78 affects the proliferation of CRC cells by regulating cell cycle progression and apoptosis. Notably, previous study reported that downregulation of GRP78 inhibited CRC cell growth and induced G1 cell cycle arrest by regulating G1/S transition-related cyclins and CDK proteins (14). Therefore, the molecular mechanism underlying the proliferation inhibition by GRP78 knockdown through inducing $\mathrm{S}$ phase arrest in CRC cells need further investigation in our further study.

Pathways which are driven by GRP78 in carcinogenesis remain largely unclear. It has been shown that AKT-ERK signaling pathway is critically involved in the regulation of proliferation and survival of tumor cells in a variety of types of cancers (21-23). Given that GRP78 regulates the activity of AKT-ERK, we examined whether AKT and ERK signaling pathways are involved in GRP78-mediated CRC cell proliferation. We found that knockdown of GRP78 reduces the phosphorylation of AKT and ERK. This result was consistent with the finding that deletion of GRP78 can suppress the AKT activation and tumorigenesis (24). Similar result was observed in astrocytoma that GRP78 expression is responsible for the promotion of cell proliferation by activating ERK $1 / 2$ and 

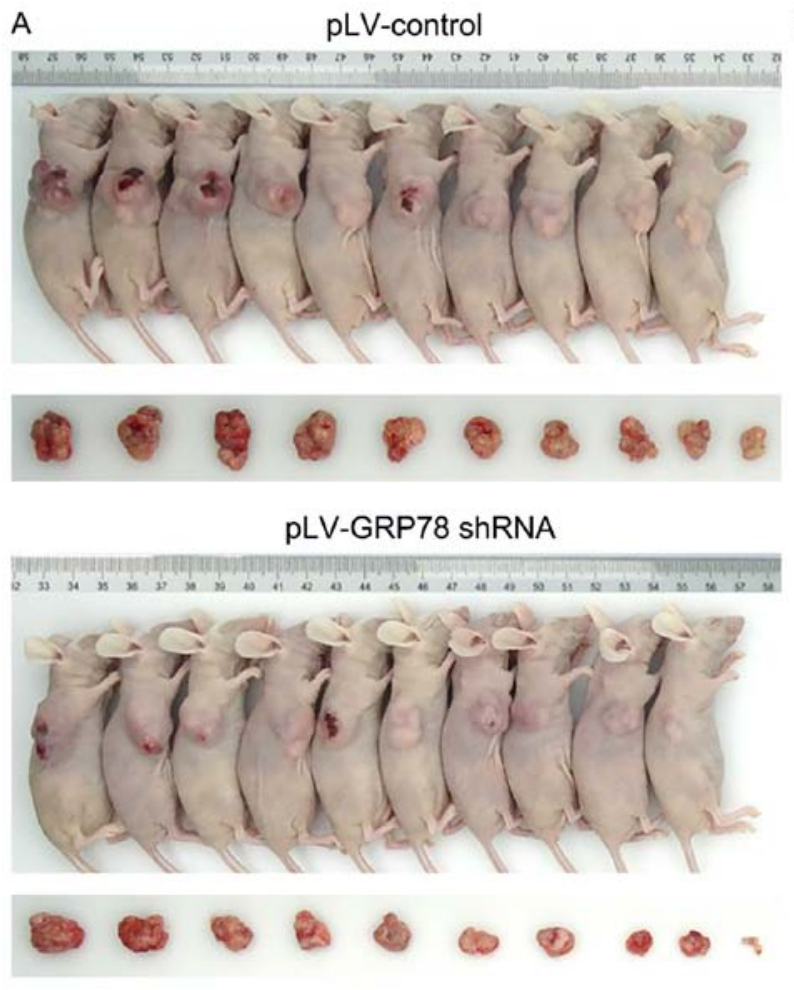
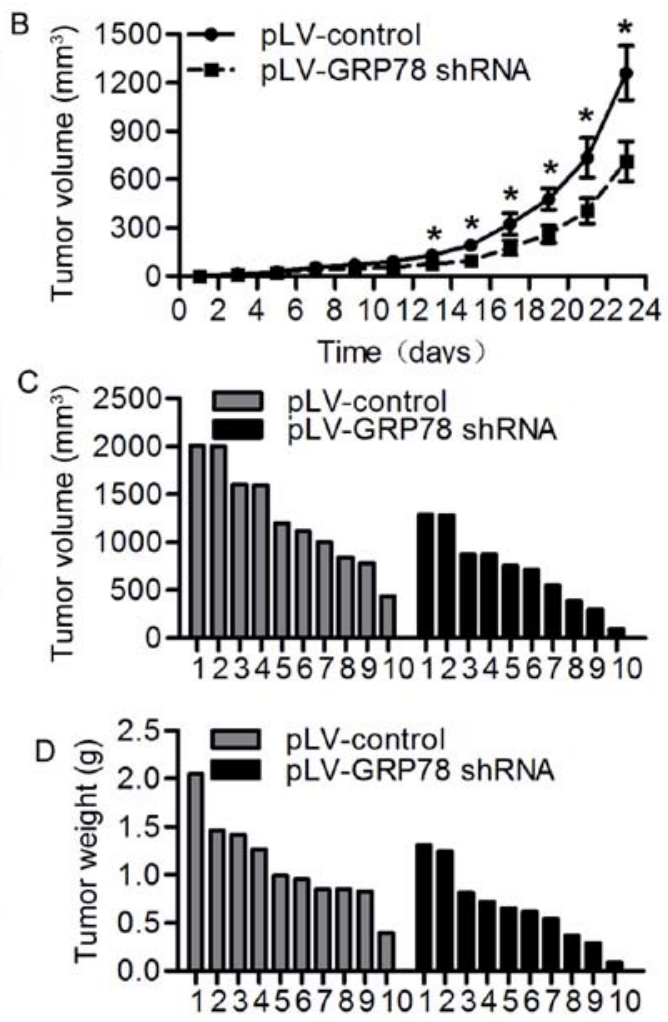

Figure 7. Inhibition of GRP78 suppresses tumor growth in vivo. Tumor formation in nude mice 6 weeks after injection with RKO cells transfected with pLV-GRP78 shRNA or pLV-control. (B) Growth curves of generated tumors by RKO cells transfected with pLV-GRP78 shRNA or pLV-control. Volumes of the tumors were measured every 2 days. Error bars, $\mathrm{SD}\left(\mathrm{n}=10 ;{ }^{*} \mathrm{P}<0.05\right.$ compared with control). (C) Volumes of the generated tumors by RKO cells transfected with pLV-GRP78 shRNA or pLV-control 6 weeks after the initial injection. (D) Weights of the generated tumors by RKO cells transfected with pLV-GRP78 shRNA or pLV-control 6 weeks after the initial injection.

AKT signaling pathways (13). We also observed that GRP78 blockade differentially affected the phosphorylation of AKT and ERK with a stronger inhibition to AKT than ERK, which may be partially explained by the finding that cell surface GRP78 is an upstream regulator of PI3K/AKT pathway, but not MAPK signaling pathway, and directly forms a complex with the PI3K subunit (27). The finding that AKT blocks ERK signaling pathway through inhibition of Raf may also contribute to the weak inhibition of GRP78 to phosphorylation of ERK in RKO cells (22).

It is unclear how GRP78 regulates AKT-ERK signaling pathway. Although primarily located in the ER lumen, GRP78 can appear on tumor cell surface and ER stress upregulates GRP78 expression on the cell surface. It has been reported that CRC cells (HT29, LOVO and SW480) have relatively high surface GRP78 expression $(27,28)$. Since GRP78 shRNA can reduce both intracellular and cell surface GRP78 expression, we speculated that both intracellular and cell surface GRP78 are involved in regulating AKT-ERK signaling pathway. Supporting this, studies have found that cell surface GRP78 interacts with activated $\alpha_{2}$ and cripto and promotes cell proliferation and survival via ERK and AKT signaling pathways $(28,29)$. In addition, blockade of cell surface GRP78 using monoclonal antibody mAb159 led to the reduction of AKT phosphorylation and the inhibition of tumor growth both in vitro and in vivo (27). It has been shown that AKT and ERK signaling pathways are involved in the induction of GRP78 (30-32). However, we found that silencing GRP78 attenuates the phosphorylation of AKT and ERK, suggesting a positive feedback between GRP78 and AKT-ERK signaling pathway. In addition, we also observed that GRP78 knockdown enhanced apoptosis and reduce the number of cells colonies after the cells were treated with 5-FU. A previous study also showed that GRP78 knockdown enhances apoptosis via the downregulation of oxidative stress and Akt pathway after epirubicin treatment in colon cancer DLD-1 cells (33). We speculate that GRP78 can be considered as a novel predictor of responsiveness to chemotherapy in CRC treatment. However, the precise molecular mechanism remains to be investigated in our further study.

The present study findings should be elucidated with consideration of its limitations. CRC patients usually also receive radiotherapy if surgery is impossible. Radiotherapy can induce cell apoptosis, helping to shrink the volume of tumors and improve the survival rate of CRC patients. However, the present study does not show whether GRP78 knockdown, along with radiation, would had a better effect on inhibiting CRC cell proliferation and promoting apoptosis. Thus, we could not precisely evaluate the potential therapeutic role of GRP78 in the treatment of CRC patients. This aspect should be explored in our future experiments.

In conclusion, our findings demonstrated that GRP78 contributes to the proliferation and tumorigenesis of CRC via AKT and ERK pathways in vitro and in vivo. GRP78 plays an important role in CRC development and progression. A better understanding of the molecular mechanism of GPR78 in CRC 
development and progression would provide novel therapeutic targets for the treatment of CRC patients.

\section{Acknowledgements}

The present study was supported by grants from the National Natural Science Foundation of China (nos. 81201947, 81502388 and 81202142), the Natural Science Foundation of Shandong, China (ZR2009CM014), and the Excellent Young Scientist Foundation of Shandong Province, China (no. 2006BSB14001).

\section{References}

1. Luo YX, Chen DK, Song SX, Wang L and Wang JP: Aberrant methylation of genes in stool samples as diagnostic biomarkers for colorectal cancer or adenomas: A meta-analysis. Int J Clin Pract 65: 1313-1320, 2011.

2. Jemal A, Siegel R, Ward E, Hao Y, Xu J and Thun MJ: Cancer statistics, 2009. CA Cancer J Clin 59: 225-249, 2009.

3. Dashwood RH: Early detection and prevention of colorectal cancer (Review). Oncol Rep 6: 277-281, 1999.

4. Li J and Lee AS: Stress induction of GRP78/BiP and its role in cancer. Curr Mol Med 6: 45-54, 2006.

5. Zhang Y, Liu R, Ni M, Gill P and Lee AS: Cell surface relocalization of the endoplasmic reticulum chaperone and unfolded protein response regulator GRP78/BiP. J Biol Chem 285: 15065-15075, 2010.

6. Rao RV, Peel A, Logvinova A, del Rio G, Hermel E, Yokota T, Goldsmith PC, Ellerby LM, Ellerby HM and Bredesen DE: Coupling endoplasmic reticulum stress to the cell death program: Role of the ER chaperone GRP78. FEBS Lett 514: 122-128, 2002

7. Takada K, Hirose J, Yamabe S, Uehara Y and Mizuta H: Endoplasmic reticulum stress mediates nitric oxide-induced chondrocyte apoptosis. Biomed Rep 1: 315-319, 2013.

8. Pyrko P, Schönthal AH, Hofman FM, Chen TC and Lee AS: The unfolded protein response regulator GRP78/BiP as a novel target for increasing chemosensitivity in malignant gliomas. Cancer Res 67: 9809-9816, 2007.

9. Song MS, Park YK, Lee JH and Park K: Induction of glucoseregulated protein 78 by chronic hypoxia in human gastric tumor cells through a protein kinase C-epsilon/ERK/AP-1 signaling cascade. Cancer Res 61: 8322-8330, 2001

10. Daneshmand S, Quek ML, Lin E, Lee C, Cote RJ, Hawes D, Cai J, Groshen S, Lieskovsky G, Skinner DG, et al: Glucose-regulated protein GRP78 is up-regulated in prostate cancer and correlates with recurrence and survival. Hum Pathol 38: 1547-1552, 2007.

11. Zheng HC, Takahashi H, Li XH, Hara T, Masuda S, Guan YF and Takano Y: Overexpression of GRP78 and GRP94 are markers for aggressive behavior and poor prognosis in gastric carcinomas. Hum Pathol 39: 1042-1049, 2008.

12. Baptista MZ, Sarian LO, Vassallo J, Pinto GA, Soares FA and de Souza GA: Prognostic significance of GRP78 expression patterns in breast cancer patients receiving adjuvant chemotherapy. Int J Biol Markers 26: 188-196, 2011.

13. Zhang LH, Yang XL, Zhang X, Cheng JX and Zhang W: Association of elevated GRP78 expression with increased astrocytoma malignancy via Akt and ERK pathways. Brain Res 1371 23-31, 2011.

14. Lin JA, Fang SU, Su CL, Hsiao CJ, Chang CC, Lin YF and Cheng CW: Silencing glucose-regulated protein 78 induced renal cell carcinoma cell line G1 cell-cycle arrest and resistance to conventional chemotherapy. Urol Oncol 32: 29.e1-29.e11, 2014

15. Xing X, Li Y, Liu H, Wang L and Sun L: Glucose regulated protein 78 (GRP78) is overexpressed in colorectal carcinoma and regulates colorectal carcinoma cell growth and apoptosis. Acta Histochem 113: 777-782, 2011.
16. Li Z, Zhang L, Zhao Y, Li H, Xiao H, Fu R, Zhao C, Wu H and Li Z: Cell-surface GRP78 facilitates colorectal cancer cell migration and invasion. Int J Biochem Cell Biol 45: 987-994, 2013.

17. Roller C and Maddalo D: The Molecular chaperone GRP78/BiP in the development of chemoresistance: Mechanism and possible treatment. Front Pharmacol 4: 10, 2013.

18. Misra UK, Deedwania R and Pizzo SV: Binding of activated alpha2-macroglobulin to its cell surface receptor GRP78 in 1-LN prostate cancer cells regulates PAK-2-dependent activation of LIMK. J Biol Chem 280: 26278-26286, 2005.

19. Chen M, Zhang Y, Yu VC, Chong YS, Yoshioka T and Ge R: Isthmin targets cell-surface GRP78 and triggers apoptosis via induction of mitochondrial dysfunction. Cell Death Differ 21: 797-810, 2014.

20. Fu R, Yang P, Wu HL, Li ZW and Li ZY: GRP78 secreted by colon cancer cells facilitates cell proliferation via PI3K/Akt signaling. Asian Pac J Cancer Prev 15: 7245-7249, 2014

21. Polivka J Jr and Janku F: Molecular targets for cancer therapy in the PI3K/AKT/mTOR pathway. Pharmacol Ther 142: 164-175, 2014.

22. Roux PP and Blenis J: ERK and p38 MAPK-activated protein kinases: A family of protein kinases with diverse biological functions. Microbiol Mol Biol Rev 68: 320-344, 2004.

23. Fruman DA and Rommel C: PI3K and cancer: Lessons, challenges and opportunities. Nat Rev Drug Discov 13: 140-156, 2014.

24. Fu Y, Wey S, Wang M, Ye R, Liao CP, Roy-Burman P and Lee AS: Pten null prostate tumorigenesis and AKT activation are blocked by targeted knockout of ER chaperone GRP78/BiP in prostate epithelium. Proc Natl Acad Sci USA 105: 19444-19449, 2008.

25. Li Z and Li Z: Glucose regulated protein 78: A critical link between tumor microenvironment and cancer hallmarks. Biochim Biophys Acta 1826: 13-22, 2012.

26. Lee AS: GRP78 induction in cancer: Therapeutic and prognostic implications. Cancer Res 67: 3496-3499, 2007.

27. Liu R, Li X, Gao W, Zhou Y, Wey S, Mitra SK, Krasnoperov V, Dong D, Liu S, Li D, et al: Monoclonal antibody against cell surface GRP78 as a novel agent in suppressing PI3K/AKT signaling, tumor growth, and metastasis. Clin Cancer Res 19: 6802-6811, 2013.

28. Misra UK, Deedwania R and Pizzo SV: Activation and crosstalk between Akt, NF-kappaB, and unfolded protein response signaling in 1-LN prostate cancer cells consequent to ligation of cell surface-associated GRP78. J Biol Chem 281: 13694-13707, 2006.

29. Kelber JA, Panopoulos AD, Shani G, Booker EC, Belmonte JC, Vale WW and Gray PC: Blockade of Cripto binding to cell surface GRP78 inhibits oncogenic Cripto signaling via MAPK/PI3K and Smad2/3 pathways. Oncogene 28: 2324-2336, 2009.

30. Zhang LJ, Chen S, Wu P, Hu CS, Thorne RF, Luo CM, Hersey P and Zhang XD: Inhibition of MEK blocks GRP78 up-regulation and enhances apoptosis induced by ER stress in gastric cancer cells. Cancer Lett 274: 40-46, 2009.

31. Feng C, He K, Zhang C, Su S, Li B, Li Y, Duan CY, Chen S, Chen R, Liu Y, et al: JNK contributes to the tumorigenic potential of human cholangiocarcinoma cells through the mTOR pathway regulated GRP78 induction. PLoS One 9: e90388, 2014.

32. Gray MJ, Mhawech-Fauceglia P, Yoo E, Yang W, Wu E, Lee AS and Lin YG: AKT inhibition mitigates GRP78 (glucose-regulated protein) expression and contribution to chemoresistance in endometrial cancers. Int J Cancer 133: 21-30, 2013.

33. Chang YJ, Huang YP, Li ZL and Chen CH: GRP78 knockdown enhances apoptosis via the down-regulation of oxidative stress and Akt pathway after epirubicin treatment in colon cancer DLD-1 cells. PLoS One 7: e35123, 2012. 